\title{
Determination of the Molecular Weight of Neuronectin, A Conditioned Medium-Derived, Substrate-Binding Neurite-Extension Factor: Comparison with Laminin Using Radiation-Inactivation Analysis
}

\author{
Michael D. Coughlin, ${ }^{\star}$ Ashok K. Grover, ${ }^{*}$ and Chan Y. Jung $\dagger$ \\ *Department of Neurosciences, McMaster University, Hamilton, Ontario L8N 3Z5, Canada, and \\ $\nmid$ Biophysics Laboratory, Veterans Administration Medical Center, State University of New York at Buffalo, Buffalo, \\ New York 14215
}

Neurite outgrowth from a wide variety of peripheral neurons is stimulated and may be directed in culture by a substrate-binding factor(s) derived from medium conditioned over numerous types of cells. This factor, or family of factors, which we shall call neuronectin by reason of its ability to serve as an attachment molecule for neurons, has been studied by target-size analysis using radiation inactivation. The radiation-inactivation method has the unique advantage of providing a means for determining the actual functional size of a biologically active molecule irrespective of its state of purification. By this method, the functional size of the major neurite outgrowth-promoting activity (neuronectin) from mouse heart cell conditioned medium has been found to be $350,000 \mathrm{Da}$. While neuronectin has not yet been purified, determination of the actual functional size provides a framework within which possible models must fit. Thus, although neurite outgrowth-promoting activity in this system is found to be associated with a complex containing laminin, fibronectin, heparan sulfate proteoglycan, and other extracellular matrix molecules, the total size of the functional molecule or molecular complex serving as the major source of activity is limited to 350,000 Da. Consequently, our results suggest that neuronectin from mouse heart cell-conditioned medium is different from laminin $\left(M_{\mathrm{r}} \sim \mathbf{9 0 0 , 0 0 0}\right)$, a molecule that also exhibits neurite-promoting activity. In addition to the difference in molecular size, neuronectin and laminin differ in that laminin, unlike neuronectin, gives rise to toxic or inhibitory products when exposed to high-energy radiation.

Neurite extension from a wide variety of peripheral neurons is stimulated and directed in culture by a substratc-binding, conditioned medium-derived factor(s) that has been partially characterized in a number of laboratories and is known variously as substrate-bound HCM (heart-cell conditioned medium) (Collins, 1978; Dribin and Barrett, 1980), PNPF (polyornithinebound neuritc promoting factor) (Adler and Varon, 1980), and CMF (conditioned medium factor) (Coughlin et al., 1981). A similar, high-molecular-weight factor isolated from bovine endothelial cells has been characterized as a heparan sulfate pro-

\footnotetext{
Received Feb. 21, 1985; revised Nov. 7, 1985; accepted Nov. 25, 1985.

We would like to thank Drs. J. Adler and E. Kempner for their constructive comments on the manuscript. We thank M. Collins, S. Samson, and C. J. Berenski for excellent technical assistance and N. Plumb for typing the manuscript. This work was supported by grants from the Medical Research Council of Canada, the Dysautonomia Foundation, Inc., and E. I. DuPont de Nemours and Co. to M.D.C., from the Heart and Stroke Foundation of Ontario to A.K.G., and from the NIH (AM-13376) to C.Y.J.

Correspondence should be addressed to Dr. Coughlin, Department of Neurosciences, McMaster University, 1200 Main Street West, Hamilton, Ontario L8N 3Z5, Canada.

Copyright $(1986$ Society for Neuroscience $0270-6474 / 86 / 061553-07 \$ 02.00 / 0$
}

teoglycan (HeSPG) or HeSPG-associated molecule (Lander et al., 1982; Matthew and Patterson, 1983). For reviews of these activities, see Berg (1984) and Coughlin (1984).

The conditioned medium factor from mouse heart cells stimulates neurite extension from peripheral neurons, promotes shortterm survival of mouse embryo sympathetic, parasympathetic, and nodose sensory neurons but not of mouse dorsal root sensory neurons, and increases specific activity of neurotransmittersynthesizing enzymes in the superior cervical sympathetic ganglion (Coughlin and Collins, 1985; Coughlin et al., 1981). The factor appears to be a general requirement for neurite extension in culture, since antiserum to the factor reversibly blocks NGFstimulated neurite extension without inhibiting NGF-stimulated biochemical development (Coughlin and Kessler, 1982). Activity is destroyed by trypsin and by heat but not by collagenase, testicular hyaluronidase, chondroitinase $\mathrm{ABC}$, or neuraminidase (Coughlin et al., 1981). This factor possesses, therefore, many of the characteristics of the factor we have described in the embryonic mouse salivary gland that stimulates and directs parasympathetic (Coughlin, 1975) and sympathetic (Coughlin et al., 1978) nerve development and that appears to be a protein or polypeptide active as a molecule bound within the extracellular matrix of the gland (Coughlin and Rathbone, 1977). Thus, the conditioned-medium activity may be similar to, or serve as a model of, a substrate factor that patterns nerve development in vivo.

The conditioned-medium factor has been partially purified by ion-exchange and gel-filtration chromatography in a number of different systems. In all systems studied, the activities are elutcd from a DEAE-agarose column as acidic molecules (Collins and Lee, 1984; Coughlin et al., 1981; Lander et al., 1983; Manthorpe et al., 1981) and migrate through a $2 \%$ agarose (CL2B) molecular sieve column at an apparent molecular weight, assuming a globular configuration, of approximately 5 million (Collins and Lee, 1984; Coughlin and Kessler, 1982; Lander et al., 1982). Despite the many common characteristics, it is not yet known if these various factors are, in fact, the same molecule, or if they represent different molecules exhibiting similar activities. Nevertheless, in light of these similarities, and by analogy with other extracellular molecules mediating cell adhesion, we shall refer to the substrate-binding, conditioned-medium-derived neurite-promoting factor(s) as neuronectin.

Recently, it has been reported that the extracellular matrix molecule laminin possesses a similar capacity for eliciting neurite extension (Baron van Evercooren et al., 1982; Manthorpe et al., 1983). The possibility that neuronectin and laminin may be identical has been suggested in several reports (Davis et al., 1984; Lander et al., 1984, 1985). In contrast, reports by Manthorpe et al. (1983) and Edgar et al. (1984) using antisera against laminin (anti-laminin) or against fragments of laminin with neu- 
rite extension-stimulating activity suggest that laminin is different from the conditioned-medium factor, since anti-laminin blocks the neurite-promoting activity of laminin but not that of the conditioned-medium factor.

To obtain an estimate of the size of neuronectin and to compare it with laminin, both laminin and the partially purified neuronectin were subjected to target-size analysis using radiation inactivation. The utilization of ionizing radiation as a method for determining functional molecular size (Lea, 1946) has gained wide acceptance as a tool in molecular characterization (see reviews by Jung, 1984; Kempner and Haigler, 1985; Kempner and Schlegel, 1979). The technique makes use of the principle that exposure of a polymer to high-energy radiation results in random damage to the molecular structure with consequent loss of biologic function. Since energy is transferred throughout the polymer from the site of the primary "hit," the resulting random damage insures that activity anywhere in the covalently linked structure is destroyed. Targets are not "partially" damaged. For example, a primary ionization event occurring anywhere within the prothrombin molecule results in the loss of thrombin activity, even though the thrombin domain occupies only half the molecule (Aronson and Preiss, 1962). The key advantage of the technique is that it does not require purification or isolation of the molecule under study. Functional activity is itself the measure utilized, and analysis of the loss of activity due to radiation exposure provides a reliable estimate of the size of the active structure. The larger the structure, the greater the probability of its being "hit" by ionizing radiation, that is, according to Poisson probability,

$$
A=A_{0} e^{-m D}
$$

where $A_{0}$ is the initial activity; $m$, the molecular mass of the target possessing activity; and $A$, the activity after a radiation dose $D$. From this relationship, the molecular weight $\left(M_{\mathrm{r}}\right)$ of the target can be derived and expressed as

$$
M_{\mathrm{r}}=\left(6.3 \times 10^{11}\right) / D_{37}
$$

where $D_{37}$ is the dosage in rads at which activity is reduced to $37 \%$ of initial activity (for derivations see Jung, 1984; Kempner and Haigler, 1985). This equation is remarkably close to the empirically derived fromula of Kepner and Macey (1968): MW = $\left(6.4 \times 10^{11}\right) / D_{37}$. Temperature, however, has a profound effect on the effective radiation dose received by samples (Kempner and Haigler, 1982). Although the Blue Cellophane method of determining radiation dose corrects for temperature differences (Jung, 1984), any remaining possible difficulties can be bypassed by using a calibration curve constructed from marker proteins of known molecular weights (Lo et al., 1982). The technique can be employed to determine molecular sizes at least over the range from 10,000 to $10,000,000 \mathrm{Da}$ and has proved especially useful in characterizing receptors and other membrane proteins that are difficult to isolate (Jung, 1984; Kempner and Haigler, 1985). Proteins studied by this technique include the ACh receptor in membrane preparations of Torpedo electric organ and cat denervated muscle (Lo et al., 1982), the slow inward calcium channel (Venter et al., 1983), the insulin receptor in rat liver plasma membranes (Harmon et al., 1980, 1981), the epidermal growth factor receptor in human carcinoma membranes (Kempner and Haigler, 1985), and the enkephalin receptor in lyophilized hybrid neuroblastoma-glioma cells (McLawhon et al., 1983).

Comparison of size determinations of over 30 enzymes and receptors made by means of radiation inactivation analysis with the "accepted" molecular weights for those proteins suggests that the agreement between target and molecular size is within approximately 14\% (Kempner and Schlegel, 1979).

Target-size analysis of the partially purified neuronectin from mouse heart cell conditioned medium indicates a molecular size for the active structure of approximately $350 \mathrm{kDa}$. In contrast, the molecular size of laminin determined by this method is the same as that found by other methods, approximately $900 \mathrm{kDa}$. These findings suggest that neuronectin, the neurite-promoting factor from conditioned medium, is different from laminin.

\section{Materials and Methods}

\section{Preparation of neuronectin and laminin}

The conditioned-medium factor (neuronectin) was used as the partially purified fraction from mouse heart cell conditioned medium. Details of the isolation have been previously reported (Coughlin et al., 1981). Briefly, embryonic mouse heart cells were grown to confluency in roller culture bottles in Nutrient Mixture F1 2 containing $10 \%$ fetal calf serum and antibiotics (F12FCS10; Grand Island Biologicals). Medium was changed every $2-3 \mathrm{~d}$, and bottles were gassed with a mixture of $5 \% \mathrm{CO}_{2}$ in air. Spent medium to be used as a source of the factor was stored frozen at $-20^{\circ} \mathrm{C}$. Neuronectin was isolated from 2-3 liters of conditioned medium passed through a DEAE-agarose column (DEAE-BioGel A, BioRad), total bed volume being $30 \mathrm{ml}$. The column was washed with $0.15 \mathrm{M} \mathrm{NaCl}$ in $10 \mathrm{~mm}$ sodium phosphate, $\mathrm{pH} 7.2$ (PBS) until UV absorbance at $280 \mathrm{~nm}$ fell below 0.01 and was eluted with $0.4 \mathrm{M} \mathrm{NaCl}$ in $10 \mathrm{~mm}$ sodium phosphate, pH 7.2. The eluate (approximately $50 \mathrm{ml}$ ) was concentrated by dialysis against sucrose, dialyzed against $0.1 \mathrm{M}$ $\mathrm{NaCl}$ in $10 \mathrm{~mm}$ HEPES ( $n$-2-hydroxyethyl piperazine- $N^{\prime}$-2-ethanesulfonic acid; Sigma), $\mathrm{pH} 7.4$, and sterilized by pressure filtration through a $0.2 \mu \mathrm{m}$ pore-size polycarbonate filter. The resulting fraction $(10 \mathrm{ml})$ of neuronectin was approximately $1 \mathrm{mg}$ protein $/ \mathrm{ml}$ with an activity of $300-500 \mathrm{units} / \mathrm{mg}, 1$ unit being that concentration of neuronectin that promotes half-maximal survival in the standard bioassay (see below). Neuronectin could be stored at $5^{\circ} \mathrm{C}$ for at least a month with no appreciable loss of activity.

Laminin was obtained commercially (BRL; Gibco) as a solution of $1 \mathrm{mg}$ protein $/ \mathrm{ml}$ in isotonic saline and stored frozen at $-70^{\circ} \mathrm{C}$.

\section{Bioassay}

Assay of biological activity was as previously described (Coughlin et al., 1981). Superior cervical ganglia of mouse embryos at $14 \mathrm{~d}$ of gestation were excised, dissociated with trypsin, and plated into $35 \mathrm{~mm}$ polyornithine-coated tissue culture dishes containing serial dilutions of samples to be tested. For most assays, dilutions were made directly into plating medium (F12FCS10). For some assays, particularly those involving laminin, polyornithine-coated dishes were preincubated for 16 $\mathrm{hr}$ with serial dilutions of the sample in PBS containing $0.02 \%$ sodium azide. Dishes were subsequently washed twice with PBS and medium added. Neuronal survival of duplicate dishes was estimated at $20 \mathrm{hr}$ of culture from counts of phase-bright cell bodies with processes greater than one cell diameter determined on $2 \%$ of the culture dish surface. Although in other systems survival does not necessarily imply neurite extension (Varon et al., 1979), under the conditions of this assay survival and neurite extension are equivalent: Embryonic day 14 SCG neurons that do not put out processes do not survive to $20 \mathrm{hr}$ in culture. For radiation-inactivation analysis, half-maximal activity for each sample was determined from dose-response curves.

\section{Radiation-inactivation, target-size analysis}

Radiation-inactivation analysis was carried out as described previously (Venter et al., 1983). Neuronectin, laminin, and enzyme standards were frozen in aluminum trays at a depth of no more than $2 \mathrm{~mm}$ by immersion in liquid nitrogen. Frozen samples were stored at $-70^{\circ} \mathrm{C}$ and subsequently irradiated at -45 to $-65^{\circ} \mathrm{C}$ with a $0.5 \mathrm{~mA}$ beam of $1.5 \mathrm{MeV}$ electrons produced by a Van de Graff generator. Radiation doses were measured at the sample irradiation temperature using Blue Cellophane (Jung, 1984). Irradiated samples were stored at $-70^{\circ} \mathrm{C}$ until assayed. Control samples were treated exactly in the same manner but not irradiated. Enzyme standards (Sigma) used for molecular-weight calibration were pyruvate kinase, $M_{\mathrm{r}} \sim 224,000$ (Lo et al., 1982); yeast alcohol dehydrogenase, $M_{\mathrm{r}} \sim 160,000$ (Lo et al., 1982); and galactose oxidase, $M_{\mathrm{r}} \sim 68,000$ (Kosman et al., 1974). Enzymes were assayed at $37^{\circ} \mathrm{C}$ by the published methods (Kosman et al., 1974; Racker, 1950; Valentine and Tanka, 1966). The plots of $\log$ percent residual activity versus radiation dose in megarads (Mrad) were linear. Slopes of these plots were computed both for standards and for neuronectin and/or laminin. 


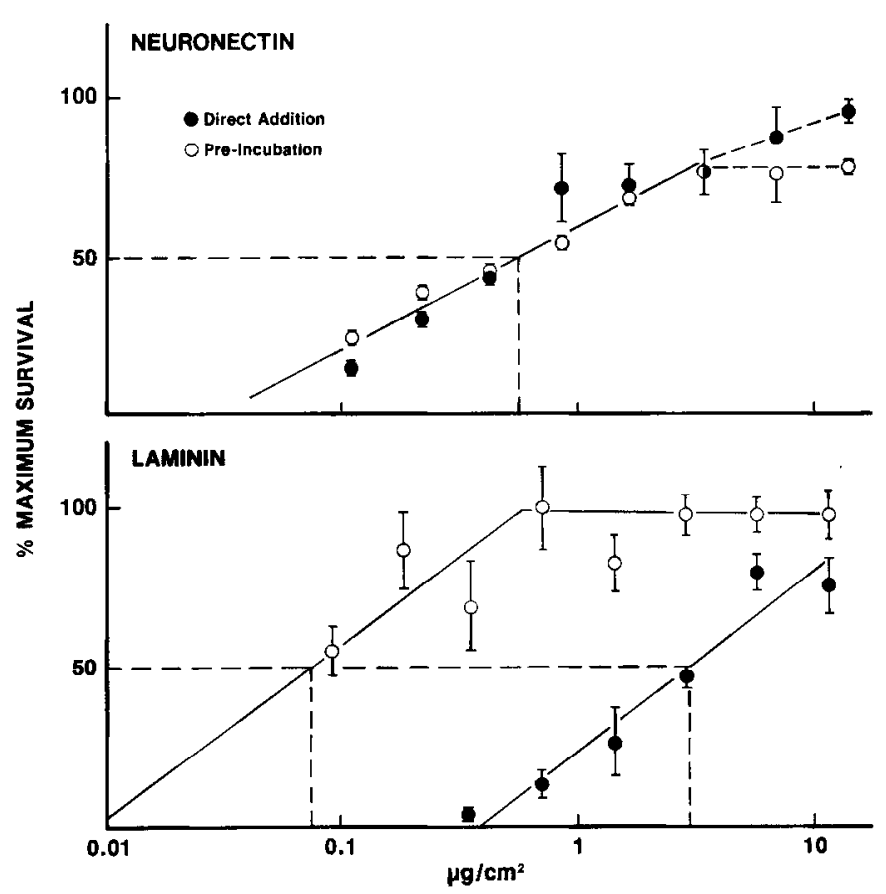

Figure 1. Comparison of the dose-response curves of neuronectin and laminin. Factors were either serially diluted directly in culture medium and assayed in PORN-coated $35 \mathrm{~mm}$ culture dishes (filled circles) or serially diluted in PBS and preincubated $16 \mathrm{hr}$ in POKN-coated tissue culture dishes that were subsequently washed and assayed (open circles). Concentrations are presented on a log scale as total factor protein present in $2 \mathrm{ml}$ medium or buffer relative to the area of the culture dish. Dissociated embryonic day 14 mouse sympathetic neurons ( 20,000/dish) were plated in medium with no added NGF. Survival was determined by counts of process-bearing phase-bright cells at $20 \mathrm{hr}$ of culture. Each value represents the mean and SEM of two separate counts on duplicate cultures. Half-maximal activity for neuronectin under both conditions was $0.6 \mu \mathrm{g} / \mathrm{cm}^{2}$. For laminin, half-maximal activity by preincubation was $0.075 \mu \mathrm{g} / \mathrm{cm}^{2}$ and by direct addition, $3 \mu \mathrm{g} / \mathrm{cm}^{2}$.

\section{Statistics}

Data are presented as means and SD, from several experiments where applicable. Linear regression was by the least-squares methods using the Minitab program on a Hewlett-Packard HP3000 computer. Bestfit curves using nonlinear regression were estimated by means of a BMDP program.

\section{Results}

\section{Bioassay conditions}

To establish a bioassay appropriate to the radiation-inactivation method, two different methods for assaying the neurite-promoting activity were examined. It has been reported that the neurite-promoting activity from conditioned medium binds to the culture substrate (Collins, 1978) and that culture dishes preincubated with serial dilutions of conditioned medium or the partially purified activity can be used for a quantitative bioassay (Adler and Varon, 1980; Black et al., 1979; Coughlin et al., 1981). For any lot of the partially purified factor, neuronectin added directly to serum-containing medium exhibits the same specific activity as that achieved by preincubating dishes with the factor in PBS (Fig. 1a). In both cases, $1 \mathrm{U}$ of activity corresponded to a concentration of approximately $3 \mu \mathrm{g} /$ $\mathrm{ml}$, or $0.6 \mu \mathrm{g} / \mathrm{cm}^{2}$. In contrast, laminin added directly to the serum-containing medium exhibits only $1 / 40$ of the activity achieved by preincubating dishes with a solution of laminin in PBS (Fig. 1b). With preincubation in PBS, 1 unit of laminin

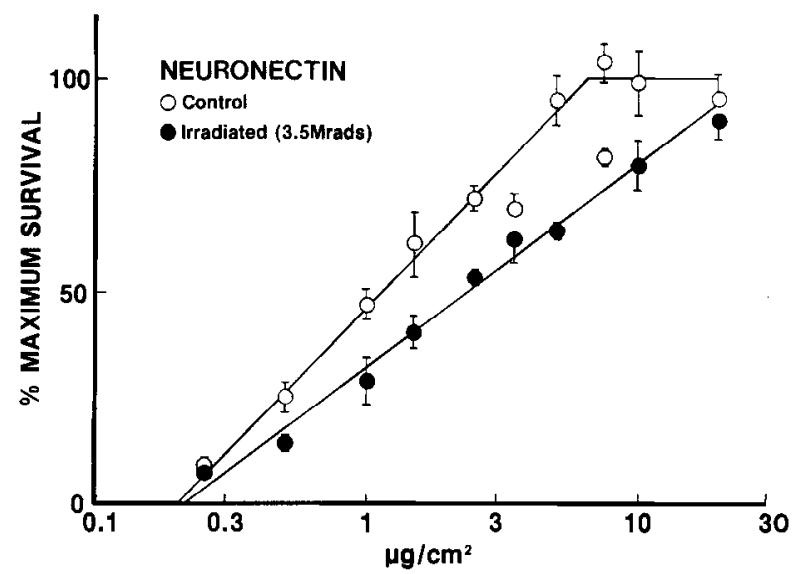

Figure 2. Effect of radiation inactivation on the dose-response curve of neuronectin. Neuronectin frozen in aluminum trays was exposed to $3.52 \mathrm{Mrad}$ of high-cnergy radiation and assayed for biological activity at concentrations between 0.25 and $20 \mu \mathrm{g} / \mathrm{cm}^{2}(1.25-100 \mu \mathrm{g} / \mathrm{ml})$ as detailed in Figure 1. Each value represents the mean and SEM of four replicate cultures. Half-maximal activity determined by the dose-response curve for the untreated sample $(O)$ was $1.1 \mu \mathrm{g} / \mathrm{cm}^{2}$ and for the sample exposed to $3.52 \mathrm{Mrad}(0), 2.4 \mu \mathrm{g} / \mathrm{cm}^{2}$.

activity was achieved at $0.075 \mu \mathrm{g} / \mathrm{cm}^{2}$, whereas direct addition required $3 \mu \mathrm{g} / \mathrm{cm}^{2}$.

Exposure of neuronectin to inactivating radiation, while decreasing specific biological activity, did not prevent neuronectin from exhibiting maximum activity at higher concentrations (Fig. 2). Therefore, radiation inactivation simply shifts the dose-response curve of neuronectin. The effect on laminin appears, however, to be quite different, as will be seen below.

\section{Radiation inactivation of enzyme standards}

Marker proteins of known molecular weights were used to construct a calibration curve. Galactose oxidase $\left(M_{\mathrm{r}} \sim 68,000\right)$, yeast alcohol dehydrogenase $\left(M_{\mathrm{r}} \sim 160,000\right)$, and pyruvate kinase $\left(M_{\mathrm{r}} \sim 224,000\right)$ were frozen, exposed to high-energy radiation, and assayed for remaining activity. Combined results of four experiments are shown in Figure 3. Analysis of the data assumes a single-hit, single-target model with simple exponential decay in activity. Average deviation of the slopes (or $D_{37}$ ) was approximately $6 \%$.

\section{Molecular-size estimation of neuronectin}

To determine the molecular size of neuronectin, samples were exposed to a graded series of radiation doses as described above. Each sample was assayed for remaining activity by dose-response curve of dilutions added directly to culture medium. The combined results of four separate experiments arc prescnted in Figure 4 , where the $\log$ residual activity is plotted against radiation dose. The best-fit curve using linear regression by the least-squares method gives $2.32 \mathrm{Mrad}(\mathrm{SD}=0.12)$ as the $D_{37}$ (Table 1). As an initial approximation, direct comparison of the slopes of pyruvate kinase and neuronectin gives a molecular weight for neuronectin of approximately $350 \mathrm{kDa}$.

\section{Radiation inactivation analysis of laminin}

Since laminin promotes neurite outgrowth and has been postulated to be responsible for the activity isolated from conditioned media (Davis et al., 1984; Lander et al., 1984, 1985), it was also subjected to similar radiation-inactivation analysis. Samples of laminin were subjected to a series of radiation doses from 0.2 to $2.2 \mathrm{Mrad}$. Dilution series at $0.05-50 \mu \mathrm{g} / \mathrm{ml}(0.01-$ $10 \mu \mathrm{g} / \mathrm{cm}^{2}$ ) of these samples in PBS were preincubated in 35 $\mathrm{mm}$ culture dishes, the dishes washed and assayed for biological 
Figure 3. Radiation inactivation of enzyme standards. Pyruvate kinase $\left(M_{\mathrm{r}} \sim 224,000\right)$, yeast alcohol dehydrogenase $\left(M_{\mathrm{r}} \sim 160,000\right)$, and galactose oxidase $\left(M_{\mathrm{r}} \sim 68,000\right)$ were frozen in aluminum trays and irradiated at -45 to $-65^{\circ} \mathrm{C}$ with a $0.5 \mathrm{~mA}$ beam of $1.5 \mathrm{MeV}$ electrons from a Van de Graff generator. Radiation doses were measured using Blue Cellophane. Enzymes were assayed for remaining activity by the published methods (see Materials and Methods). Results of four separate experiments for each enzyme are presented as the log percent residual activity versus radiation dose (in Mrad). Resultant slopes from these plots are given in Table 1 .

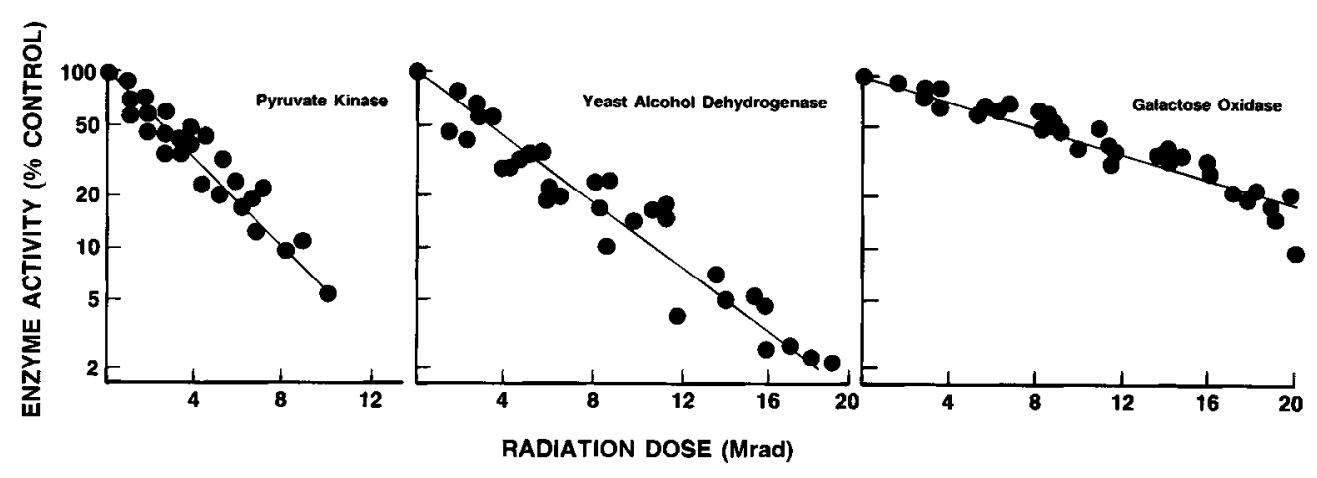

activity. All samples reached maximum activity at the higher concentrations. Residual activity was determined from halfmaximal values and is presented as the plot of $\log$ percent residual activity versus dose (Fig. 4). By the preincubation method of assay, the $D_{37}$ of laminin is $0.96 \mathrm{Mrad}$. Comparison of the slopes of laminin and pyruvate kinasc (Table 1) gives a calculated molecular weight for laminin of $882 \mathrm{kDa}$, which is essentially the same as the molecular weight of $900 \mathrm{kDa}$ determined by previous methods (Engel et al., 1981; Ott et al., 1982; Timpl et al., 1979).

As noted above, direct addition of laminin to culture medium resulted in specific activity much lower than that found with

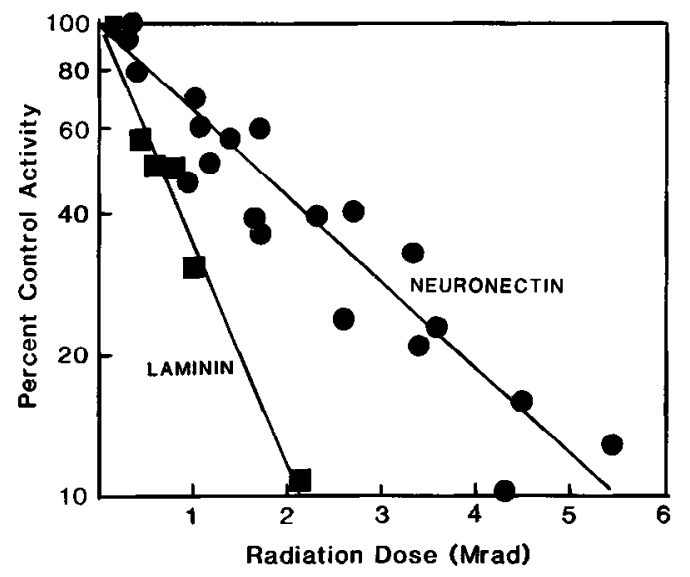

Figure 4. Radiation-inactivation, target-size analysis of neuronectin and laminin. Partially purified neuronectin (DEAE fraction) at $1 \mathrm{mg} /$

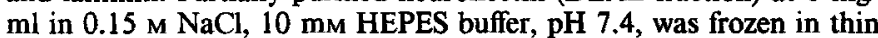
layers in aluminum trays and subjected to high-energy electron bombardment as detailed in Figure 3. Radiation-induced inactivation was determined by dose-response curve of each sample in the standard bioassay (see Fig. 2). Each point (solid circles) represents the percentage of remaining activity of treated samples of neuronectin determined by comparing half-maximal activity to that of samples not exposed to radiation. Values are presented on a semilogarithmic plot as a function of activity and of increasing radiation dosage. Lines were drawn by least-squares linear regression of the values from four separate experiments. Laminin at $1 \mathrm{mg} / \mathrm{ml}$ in $0.15 \mathrm{M} \mathrm{NaCl}, 10 \mathrm{~mm}$ Tris-Cl buffer, $\mathrm{pH}$ 7.4 , was frozen in aluminum trays, subjected to high-energy radiation, and assayed for neurite outgrowth-promoting activity by first incubating serial dilutions of samples in PBS for $16 \mathrm{hr}$ on PORN-coated tissue culture dishes. Dishes were washed twice with PBS and assayed for activity as in Figure 1. The half-maximal activity from dose-response curves was used to calculate the percentage of remaining activity. Values (solid squares) are presented on a semilogarithmic plot as a function of radiation dose, with the line drawn by least-squares linear regression. Resultant slopes and calculated molecular weights are given in Table 1. preincubation (Fig. 1). Moreover, direct addition of irradiated laminin to culture medium resulted in the appearance of inhibitory or toxic activity. Samples of laminin were subjected to a graded series of radiation doses as above and assayed for acitivity by direct addition to culture medium instead of by preincubation. Increased doses of radiation resulted not only in the loss of biological activity, but also in the appearance of inhibitory or toxic substances at the higher concentrations (Fig. 5). Nevertheless, using the activities derived from the curves in Figure 5 , the $D_{37}$ for laminin is calculated to be $0.90 \mathrm{Mrad}$ if an cxtrapolated half-maximal activity is used for the $1.15 \mathrm{Mrad}$ value. If both the 1.15 and $2.27 \mathrm{Mrad}$ values are eliminated (since they do not actually reach even half-maximal activity), the $D_{37}$ is $1.02 \mathrm{Mrad}$. These $D_{37}$ are surprisingly similar to the value found by the preincubation assay, despite the large differences in specific activity between the two methods and despite the complicating presence of inhibitory substances in the direct addition assay. Thus, by either assay, the molecular size of laminin approximates $900 \mathrm{kDa}$.

If laminin $\left(M_{\mathrm{r}}=900,000\right)$ is used as a standard along with enzymes of known molecular weight, the resulting standard curve gives a calculated molecular weight for neuronectin of $352,000 \pm$ 21,000 (Fig. 6, Table 1).

\section{Discussion}

Radiation inactivation analysis has the unique advantage of providing a means for determining the actual functional size of a biologically active molecule irrespective of the state of purification. By this method, the functional size of the major neurite outgrowth-promoting activity (neuronectin) from mouse heart cell conditioned medium has been found to be $350,000 \mathrm{Da}$. While the neurite outgrowth-promoting factor has yet to be purified, determination of the actual functional size provides a framework within which possible models must fit. Thus, these results indicate that neuronectin from mouse heart cell conditioned medium is different from laminin $\left(M_{\mathrm{r}} \sim 900,000\right)$. Consequently, although neurite outgrowth-promoting activity in this system is found associated with laminin, fibronectin, heparan sulfate proteoglycan, and other extracellular matrix molecules, most elements within the complex are not necessary for activity, since the total size of the functional molecule or molecular composite serving as the major source of activity is 350,000 .

\section{Neuronectin and laminin}

Reports that the purified molecule laminin is able to stimulate neurite outgrowth from cultured peripheral neurons (Baron van Evercooren et al., 1982; Manthorpe et al., 1983) in a manner similar to that previously described for conditioned medium (Collins, 1978; Helfand et al., 1976, 1978) raise the possibility that the activity found in conditioned medium might, in fact, 
Table 1. Molecular-weight estimation of target proteins using radiation inactivation

\begin{tabular}{lcllc} 
Protein & $\begin{array}{l}\text { Actual } M_{\mathrm{r}} \\
(\mathrm{Da})\end{array}$ & $D_{37}$ & $\begin{array}{l}\text { Slope } \\
\left(\log A / A_{0} \text { vs. dose) }\right.\end{array}$ & Predicted $M_{\mathrm{r}}$ \\
\hline Galactose oxidase & 68,000 & $12.2 \pm 0.5$ & $0.0357 \pm 0.0014$ & $57,000 \pm 2,800$ \\
Yeast alcohol dehydrogenase & 160,000 & $4.62 \pm 0.18$ & $0.0946 \pm 0.0038$ & $176,000 \pm 7,800$ \\
Pyruvate kinase & 224,000 & $3.78 \pm 0.22$ & $0.1160 \pm 0.0066$ & $220,000 \pm 18,000$ \\
Laminin & 900,000 & $0.96 \pm 0.07$ & $0.4517 \pm 0.0316$ & $899,000 \pm 64,000$ \\
Neuronectin & & $2.32 \pm 0.12$ & $0.1815 \pm 0.0102$ & $352,000 \pm 21,000$
\end{tabular}

Solutions of proteins were frozen in aluminum trays and irradiated at -45 to $-65^{\circ} \mathrm{C}$ with a $0.5 \mathrm{~mA}$ beam of $1.5 \mathrm{MeV}$ electrons at various doses as shown in Figures 3 and 4. Enzymes were assayed by published methods, while laminin and neuronectin were assayed for biological activity by dose-response determination (see Materials and Methods). Results are derived from plots of $\log$ percent residual activity versus radiation dose in megarads (Figs. 3,4$)$ and are presented as the mean $\pm \mathrm{SD}$. Predicted $M_{\mathrm{r}}$ was calculated from the standard curve fit by least-squares linear regression to the values for the slopes of the three enzymes and laminin (Fig. 6).

be laminin. Moreover, it has been reported that anti-laminin is capable of immunoprecipitating from conditioned medium the neurite-promoting activity (Davis et al., 1984; Lander et al., 1984,1985 ). Finally, there are also indications that lamininlike immunoreactivity copurifies with the conditioned mediumderived activity (Davis et al., 1984; Lander et al., 1984, 1985). Thus, there is reason to suspect that laminin might be responsible for the conditioned medium-derived activity. On the other hand, there are, in addition to the target-size differences reported here, major difficulties in ascribing the activity of the conditioned medium-derived neuronectin to laminin. Polyclonal antilaminin, while blocking laminin activity, does not block the neurite-promoting activity of neuronectin, and conversely, antiserum to the partially purified conditioned medium-derived factor (anti-neuronectin), while blocking neuronectin activity, does not block the neurite-promoting activity of laminin (Edgar et al., 1984; Manthorpe et al., 1983; personal observation). One possible explanation for these seemingly contradictory results is that both laminin and neuronectin are bound to proteoglycan. Neuronectin activity has been shown to be associated with proteoglycan during purification (Lander et al., 1982), and laminin is known to bind strongly to proteoglycan (Ott et al., 1982). It is not then surprising that the partially purified neuronectin examined in these studies does appear to include some laminin as determined by Ouchterlony double-diffusion immunoprecip-

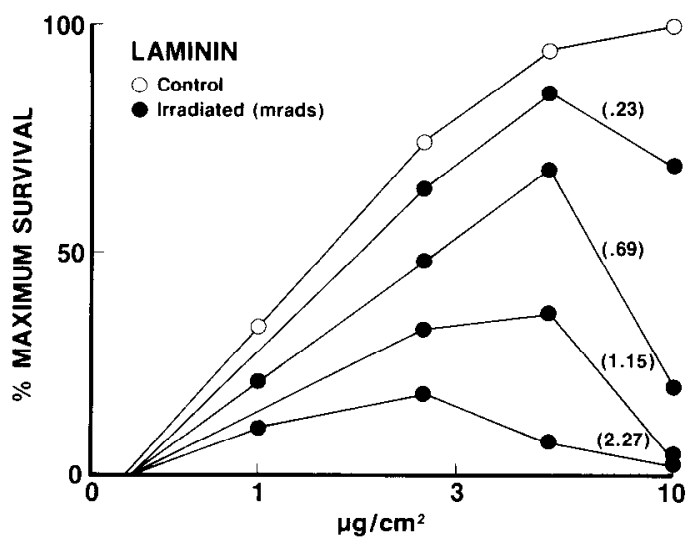

Figure 5. Effect of radiation inactivation on the dose-response curve of laminin added directly to culture medium. Laminin was subjected to radiation inactivation as in Figure 4, except that assays were performed by dilution of samples directly into the plating medium for the neurons. Values for the dose-response curves are the average of duplicate cultures. Control samples were frozen in trays and subjected to the same conditions as samples exposed to various levels of radiation (Mrad in parentheses). itation (unpublished observations). Thus, immunoprecipitation of laminin with specific antibodies would also bring down neuronectin, while the same anti-laminin would not be expected to block neuronectin activity were it, in fact, due to a molecule distinct from laminin.

Although laminin is associated with the partially purified neurite-promoting activity from conditioned medium and may contribute to that activity, radiation-inactivation analysis indicates that the major species responsible for activity is not laminin and therefore suggests that laminin and neuronectin are different molecules. Target-size analysis confirms a molecular weight for the tumor-derived laminin of approximately $900 \mathrm{kDa}$, in accord with determinations by other methods (Engel et al., 1981; Ott et al., 1982). In contrast, the molecular weight of neuronectin is approximately $350 \mathrm{kDa}$ (Fig. 3 ). One limitation of the radiation-inactivation technique is the difficulty in resolving multiple targets having the same activity (discussion in Kempner and Haigler, 1985). In the case at hand, the presence of laminin in our preparation would not detectably alter the inactivation curve

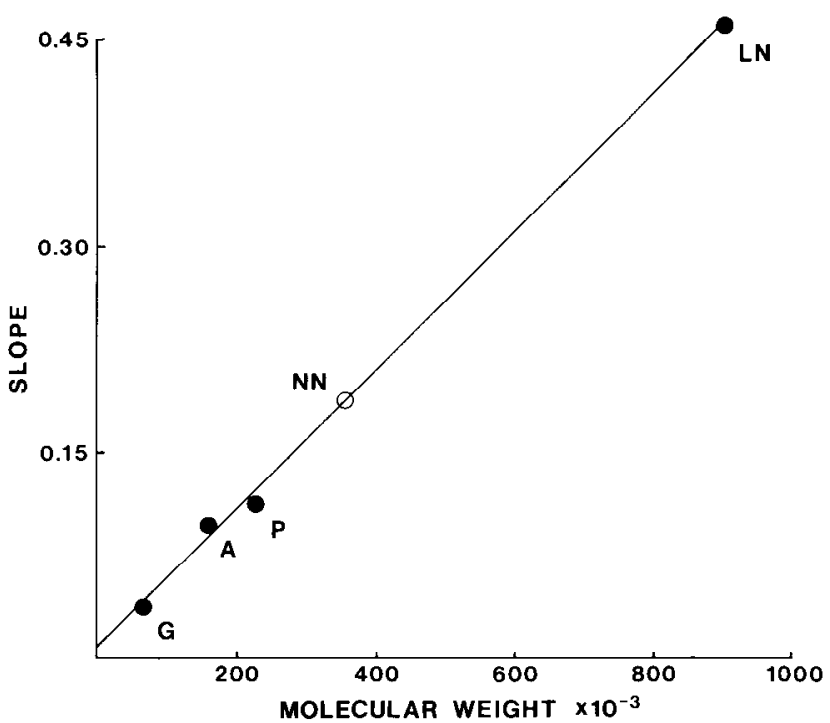

Figure 6. Radiation-inactivation molecular-weight calibration curve. Standard enzymes [galactose oxidase $(G), 68 \mathrm{kDa}$; yeast alcohol dehydrogenase $(A), 160 \mathrm{kDa}$; pyruvate kinase $(P), 224 \mathrm{kDa}$ ] and laminin $(L N), 900 \mathrm{kDa}$, were inactivated individually, their inactivation slopes determined and plotted relative to known molecular weights (data from Table 1). The line drawn by least-squares linear regression has a correlation coefficient of 0.9993 . From these data, the molecular weight of neuronectin was calculated to be $352,000 \mathrm{Da}$. Molecular-weight values similarly calculated for the other proteins are given in Table 1. 
unless it contributed more than half the initial activity. Where one target contributes most of the activity, a molecular size close to that of the major species will be observed. For two targets differing greatly in size and both contributing significantly to the activity, the activity decay curve will be biphasic. Although the data presented in Figure 4 can be fitted to a restricted range of nonlinear curves to include some contribution of laminin to total activity, the regression curve with the least residual mean sum of squares remains that of simple exponential decay.

In addition to the differences in molecular size, laminin differs from neuronectin in that laminin gives rise to inhibitory or toxic break-down products in response to high-energy radiation exposure, whereas neuronectin does not (cf. Figs. 2 and 5). This difference in response to radiation inactivation is itself an indication of fundamental differences between laminin and neuronectin. Finally, even though neurite-promoting activity of the laminin molecule may be attributable to a single fragment, fragment 3 (Edgar et al., 1984), activity under normal conditions is a property of the whole, undissociated molecule, since radiation-inactivation analysis gives as the functional entity the whole $900 \mathrm{kDa}$ molecule in which activity is destroyed by a single hit anywhere within the molccular volume. Thus, all subunits remain tightly enough bound in the functional state that the entire structure serves as a target for ionizing radiation.

While these results show a distinction between neuronectin and the tumor-derived laminin, they do not rule out the possibility that neuronectin may be similar to a subunit of laminin released free into medium by cultured cells (Davis et al., 1984). However, the latter postulate still does not account for the fact that polyclonal antibodies to laminin do not block the activity of neuronectin, and more especially that antibodies to neuronectin do not block the neurite-promoting activity of laminin. It would seem more likely that immunoprecipitation of activity and immunohistochemical staining of copurified molecules are due to the contaminating presence of laminin or to the existence of a common epitope that does not participate in the activity of neuronectin.

\section{Neuronectin and heparan sulfate proteoglycan}

The straight-line profile of the radiation inactivation curve of neuronectin (Fig. 3) suggests that activity resides in a molecule that behaves as a normal single-hit target for radiation inactivation. The observation that neuronectin exhibits the same activity whether added directly to serum-containing medium or allowed to bind to the substrate by preincubation suggests that both the strong substrate-binding property and biological activity reside in the same molecule or molecular complex.

Although radiation-inactivation analysis indicates an $M_{\mathrm{r}}$ for neuronectin of $350 \mathrm{kDa}$, it does not provide information on the composition or the form in which it is isolated. Gel filtration of activity indicates an apparent size for activity-containing material of 3-5 million (Coughlin and Kessler, 1982; Lander et al., 1982). The very large size may be due to an association of the activity with a complex of proteoglycan and other proteins (Lander et al., 1982) or to the extended shape of a molecule such as a proteoglycan, which would result in anomalous behavior during gel filtration under standard conditions. Neuronectin appears to be associated with proteoglycan, since activity is precipitated from solution by acid-albumin (unpublished observations), which has been shown to precipitate acidic proteoglycans quantitatively (Szewczyk, 1983).

Since neuronectin appears to be associated with heparan sulfate proteoglycan (Lander et al., 1982), and since the molecular weight of neuronectin $\left(M_{\mathrm{r}} \sim 350,000\right)$ is identical to that determined for the heparan sulfate proteoglycan by gel filtration in $4 \mathrm{M}$ guanidinium hydrochloride (Matthew et al., 1985), it might appear that neuronectin is, in fact, heparan sulfate proteoglycan. However, for reasons as not yet determined, radiation inactivation of glycoconjugates does not include as part of the molecular size carbohydrate moieties that are not necessary for activity (Lowe and Kempner, 1982). Since glycosylation of the active protein does not appear necessary for neuronectin-like activity (Lander et al., 1982), only the core protein of the heparan sulfate proteoglycan would be measured by target-size analysis, were it the active factor. The $M_{\mathrm{r}}$ of the heparan sulfate proteoglycan core protein has been reported to be approximately 80,000 (Matthew et al., 1985). The results presented here suggest, therefore, that neuronectin activity is not the heparan sulfate proteoglycan. Previous studies on the heparan sulfate proteoglycan have left open the question of whether neuritepromoting activity is a property of the proteoglycan itself or of an associated protein (Lander et al., 1982, 1983, 1984). The more recent evidence indicates the latter (Lander et al., 1985), in agreement with the present findings. Moreover, it has not been possible to replicate the effects of conditioned-mediumderived neuronectin with purified glycosaminoglycans, including heparin and a heparan sulfate proteoglycan (Carbonetto et al., 1983; Manthorpe et al., 1983). If anything, the purified glycosaminoglycans appear to inhibit neurite outgrowth (Carbonctto et al., 1983).

\section{Summary}

Neuronectin, the substrate-binding, neurite-promoting factor from mouse heart cell conditioned medium has a $M_{\mathrm{r}} \sim 350,000$, as determined by the radiation-inactivation method. Using similar methods, the size of laminin as assayed by its neurite-promoting properties has been confirmed to be $M_{\mathrm{r}} \sim 900,000$. These studies suggest that neuronectin differs from laminin and other model neurite-promoting factors, further confirming the multiplicity of such factors. It is, in fact, quite possible that different types of cultured cells produce different neuronectins. Such a multiplicity of neurite-promoting factors might provide a mechanism for the formation of specific axonal pathways.

\section{References}

Adler, R., and S. Varon (1980) Cholinergic neuronotrophic factors: $V$. Segregation of survival and neurite-promoting activities in heartconditioned medium. Brain Res. 188: 437-448.

Aronson, D. L., and J. W. Preiss (1962) Molecular weights of human prothrombin and thrombin by electron irradiation. Radiat. Res. 16: 138-143.

Baron van Evercooren, A., H. K. Kleinmen, S. Ohno, P. Marangos, J. P. Schwartz, and M. DuBois-Dalcq (1982) Nerve growth factor, laminin and fibronectin promote neurite outgrowth in human fetal sensory ganglia cultures. J. Neurosci. Res. 8: 179-193.

Berg, D. K. (1984) New neuronal growth factors. Annu. Rev. Neurosci 7: $149-170$

Black, I. B., M. D. Coughlin, and P. Cochard (1979) Factors regulating neuronal differentiation. Soc. Neurosci. Symp. 4: 184-204.

Carbonetto, S., M. M. Gruver, and D. C. Turner (1983) Nerve fiber growth in culture on fibronectin, collagen, and glycosaminoglycan substrates. J. Neurosci. 3: 2324-2335.

Collins, F. (1978) Induction of neurite outgrowth by a conditionedmedium factor bound to the culture substratum. Proc. Natl. Acad. Sci. USA 75: 5210-5213.

Collins, F., and M. R. Lee (1984) The spatial control of ganglionic neurite growth by the substrate-associated material from conditioned medium: An experimental model of haptotaxis. J. Neurosci. 4: 28232829.

Coughlin, M. D. (1975) Target organ stimulation of parasympathetic nerve growth in the developing mouse submandibular gland. Dev. Biol. 43: 140-158.

Coughlin, M. D. (1984) Growth factors regulating autonomic nerve development. Adv. Cell. Neurobiol. 5: 53-112.

Coughlin, M. D., and M. B. Collins (1985) Nerve growth factor (NGF)Independent development of embryonic mouse sympathetic neurons in dissociated cell culture. Dev. Biol. 110: 392-401.

Coughlin, M. D., and J. A. Kessler (1982) Antiserum to a new neuronal 
growth factor: Effects on neurite outgrowth. J. Neurosci. Res. 8: 289. 302.

Coughlin, M. D., and M. P. Rathbone (1977) Factors involved in the stimulation of parasympathetic nerve outgrowth. Dev. Biol. 61: 131139.

Coughlin, M. D., M. D. Dibner, D. M. Boyer, and I. B. Black (1978) Factors regulating development of an embryonic mouse sympathetic ganglion. Dev. Biol. 66: 513-528.

Coughlin, M. D., E. M. Bloom, and I. B. Black (1981) Characterization of a neuronal growth factor from mouse heart-cell conditioned medium. Dev. Biol. 82: 56-68.

Davis, G. E., M. Manthorpe, and S. Varon (1984) Purification of rat Schwannoma neurite promoting factor. Soc. Neurosci. Abstr. 10:38.

Dribin, L. B., and J. N. Barrett (1980) Conditioned medium enhances neurite outgrowth from rat spinal cord explants. Dev. Biol. 74: 184195.

Edgar, D., R. Timple, and H. Thoenen (1984) The heparin-binding domain of laminin is responsible for its effects on neurite outgrowth and neuronal survival. EMBO J. 3: 1463-1468.

Engel, J., E. Odermatt, A. Engel, J. A. Madri, H. Furthmayr, H. Rohde, and R. Timpl (1981) Shapes, domain organizations and flexibility of laminin and fibronectin, two multifunctional proteins of the extracellular matrix. J. Mol. Biol. 150: 97-120.

Harmon, J. T., C. R. Kahn, E. S. Kempner, and W. Schlegel (1980) Characterization of the insulin receptor in its membrane environment by radiation inactivation. J. Biol. Chem. 255: 3412-3419.

Harmon, J. T., E. S. Kempner, and C. R. Kahn (1981) Demonstration by radiation inactivation that insulin alters the structure of the insulin receptor in rat liver membranes. J. Biol. Chem. 256: 7719-7722.

Helfand, S. L., G. A. Smith, and N. K. Wessells (1976) Survival and development in culture of dissociated parasympathetic neurons from ciliary ganglia. Dev. Biol. 50: 541-547.

Helfand, S. L., R. J. Riopelle, and N. K. Wessells (1978) Non-equivalence of conditioned medium and nerve growth factor for sympathetic, parasympathetic and sensory neurons. Exp. Cell. Res. 113: 3945.

Jung, C. Y. (1984) Molecular weight determination by radiation inactivation. In Molecular and Chemical Characterization of Membrane Receptors, pp. 193-208, Liss, New York.

Kempner, E. S., and H. T. Haigler (1982) The influence of low temperature on the radiation sensitivity of enzymes. J. Biol. Chem. 257. 13297-13299.

Kempner, E. S., and H. T. Haigler (1985) Target analysis of growth factor receptors. In Growth and Maturation Factors, Vol. 3, G. Guroff, ed., pp. 149-173, Wiley, New York.

Kempner, E. S., and W. Schlegel (1979) Size determination of enzymes by radiation inactivation. Anal. Biochem. 92: 2-10.

Kepner, G. R., and R. I. Macey (1968) Membrane enzyme systems: Molecular size determinations by radiation inactivation. Biochim. Biophys. Acta 163: 188-203.

Kosman, D. J., M. J. Ettinger, R. E. Weiner, and E. J. Massano (1974) The molecular properties of the copper enzyme galactose oxidase. Arch. Biochem. Biophys. 165: 456-467.

Lander, A. D., D. K. Fujii, D. Gospodarowicz, and L. F. Reichardt (1982) Characterization of a factor that promotes neurite outgrowth: Evidence linking activity to a heparan sulfate proteoglycan. J. Cell Biol. 94: 574-585.
Lander, A. D., K. Tomaselli, A. L. Calof, and L. F. Reichardt (1983) Studies on extracellular matrix components that promote neurite outgrowth. Cold Spring Harbor Symp. Quant. Biol. 48: 611-623.

Lander, A. D., D. K. Fujii, D. Gospodarowicz, and L. F. Reichardt (1984) Neurite-outgrowth-promoting factors in conditioned media are complexes containing laminin. Soc. Neurosci. Abstr. 10: 40.

Lander, A. D., D. K. Fujii, and L. F. Reichardt (1985) Laminin is associated with the "neurite outgrowth-promoting factors" found in conditioned media. Proc. Natl. Acad. Sci. USA 82: 2183-2187.

Lea, D. E. (1946) Action of Radiations on Living Cells, Cambridge U. P., London.

Lo, M. M. S., E. A. Barnard, and J. O. Dolly (1982) Size of acetylcholine receptors in the membrane. An improved version of the radiation inactivation method. Biochemistry 21: 2210-2217.

Lowe, M. E., and E. S. Kempner (1982) Radiation inactivation of the glycoprotein, invertase. J. Biol. Chem. 257: 12478-12480.

Manthorpe, M., S. Varon, and R. Adler (1981) Neurite-promoting factor (NPF) in conditioned medium from RN22 schwannoma cultures: Bioassay, fractionation and other properties. J. Neurochem. 37: 759-767.

Manthorpe, M., E. Engvall, E. Ruoslahti, R. M. Longo, G. E. Davis, and $S$. Varon (1983) Laminin promotes neuritic regeneration from cultured peripheral and central neurons. J. Cell Biol. 97: 1882-1898.

Matthew, W. D., and P. H. Patterson (1983) The production of a monoclonal antibody that blocks the action of a neurite outgrowthpromoting factor. Cold Spring Harbor Symp. Quant. Biol. 48: 625631.

Matthew, W., R. Greenspan, A. Lander, and L. Reichardt (1985) Immunopurification and characterization of a neuronal heparan sulfate proteoglycan. J. Neurosci. 5: 1842-1850.

McLawhon, R. W., J. C. Ellory, and G. Dawson (1983) Molecular size of opiate (enkephalin) receptors in neuroblastoma-glioma hybrid cells as determined by radiation inactivation analysis. J. Biol. Chem. 258: 2102-2105.

Ott, U., E. Odermatt, J. Engel, H. Furthmayr, and R. Timpl (1982) Protease resistance and conformation of laminin. Eur. J. Biochem. 123: 63-72.

Racker, E. (1950) Crystalline alcohol dehydrogenase from baker's yeast. J. Biol. Chem. 184: 313-319.

Szewczyk, B. (1983) A sensitive staining method for detecting acidic polysaccharides in cellulose acetate and agarose gels. Anal. Biochem. 130: 60-64.

Timpl, R., H. Rohde, P. G. Robey, S. I. Rennard, J. M. Foidart, and G. R. Martin (1979) Laminin-A glycoprotein from basement membranes. J. Biol. Chem. 254: 9933-9937.

Valentine, W., and K. R. Tanka (1966) Pyruvate kinase: Clinical aspects. Methods Enzymol. 9: 468-473.

Varon, S., M. Manthorpe, and R. Adler (1979) Cholinergic neuronotrophic factors: I. Survival, neurite outgrowth and choline acetyltransferase activity in monolayer cultures from chick embryo ciliary ganglia. Brain Res. 173: 29-45.

Venter, J. C., C. M. Fraser, J. S. Schaber, C. Y. Jung, G. Bolger, and D. J. Triggle (1983) Molecular properties of the slow inward calcium channel. J. Biol. Chem. 258: 9344-9348. 